Vietnam Journal of Mechanics, VAST, Vol. 26, 2004, No 2 (103-110)

\title{
HIGHER ORDER STOCHASTIC AVERAGING METHOD FOR MECHANICAL SYSTEMS HAVING TWO DEGREES OF FREEDOM
}

\author{
NGuYen Duc Tinh \\ Mining Technical College, Quang Ninh
}

\begin{abstract}
Higher order stochastic averaging method is widely used for investigating single-degree-of-freedom nonlinear systems subjected to white and coloured random noises. In this paper the method is further developed for two-degree-of-freedom systems. An application to a system with cubic damping is considered and the second approximation solution to the Fokker-Planck (FP) equation is obtained.
\end{abstract}

\section{Introduction}

The stochastic averaging method was extended by Stratonovich (1963) and has a mathematically rigorous proof by Khasminskii (1963). At present, the stochastic averaging method (SAM) is widely used in different problems of stochastic mechanics, such as vibration, stability and reliability problems (see e.g. Ariaratnam and Tam, 1979; Bolotin, 1984; Roberts and Spanos, 1986; Zhu, 1988). It should be noted that principally only first order SAM has been applied in practice and usually to systems subject to white noise or wideband random processes. However, the effect of some nonlinear terms is lost during the first order averaging procedure. In order to overcome this insufficiency, different averaging procedures for obtaining approximate solutions have been developed (see e.g. Mitropolskii et al, 1992; Red-Horse and Spanos, 1992; Zhu and Lin, 1994; Zhu et al, 1997). Recently: a higher order averaging procedure using FP equation is developed in (Anh, 1993) and then applied to the systems having one degree of freedom under white noise and coloured noise excitations (Anh and Tinh, 1995; Tinh, 1999). In the present paper this procedure is further developed to lightly nonlinear systems subject to white noise excitations. An application to the system with cubic damping is considered.

\section{Higher approximate solutions to FP equation}

Consider the equations of motion of a mechanical system with two degrees of freedom

$$
\begin{aligned}
& \ddot{x}_{1}+c_{11} x_{1}+c_{12} x_{2}=\varepsilon f_{11}(x, \dot{x})+\varepsilon^{2} f_{12}(x, \dot{x})+\sqrt{\varepsilon} \sigma_{1} \dot{\xi}(t), \\
& \ddot{x}_{2}+c_{21} x_{1}+c_{22} x_{2}=\varepsilon f_{21}(x, \dot{x})+\varepsilon^{2} f_{22}(x, \dot{x})+\sqrt{\varepsilon} \sigma_{2} \dot{\xi}(t),
\end{aligned}
$$

where $c_{i j}(i, j=1,2), \sigma_{1}, \sigma_{2}$ are constants $\sigma_{1}>0, \sigma_{2}>0, \varepsilon$ is a small positive parameter, $f_{i j},(i, j=1,2)$ are functions of $x=\left(x_{1}, x_{2}\right)$ and $\dot{x}=\left(\dot{x}_{1}, \dot{x}_{2}\right)$. The random excitation $\dot{\xi}(t)$ is a Gaussian white noise process with unit intensity. 
Suppose that the characteristic equation of the system (2.1)

$$
D(\lambda)=\left|\begin{array}{cc}
c_{11}-\lambda & c_{12} \\
c_{21} & c_{22}-\lambda
\end{array}\right|=0
$$

has two distinct positive solutions $\omega_{1}^{2}, \omega_{2}^{2},\left(0<\omega_{1}^{2}<\omega_{2}^{2}\right)$.

Now we introduce the principal coordinates $u_{1}, u_{2}$ from the primary coordinates $x_{1}$, $x_{2}$ by the relations

$$
\begin{aligned}
& x_{1}=u_{1}+u_{2}, \\
& x_{2}=d_{1} u_{1}+d_{2} u_{2},
\end{aligned}
$$

where

$$
d_{1}=\frac{\omega_{1}^{2}-c_{11}}{c_{12}}=\frac{c_{21}}{\omega_{1}^{2}-c_{22}}, \quad d_{2}=\frac{\omega_{2}^{2}-c_{11}}{c_{12}}=\frac{c_{21}}{\omega_{2}^{2}-c_{22}} .
$$

Substituting (2.2) into (2.1) we have

$$
\begin{aligned}
& \ddot{u}_{1}+\omega_{1}^{2} u_{1}=\varepsilon F_{11}+\varepsilon^{2} F_{12}+\sqrt{\varepsilon} G_{1} \dot{\xi}(t), \\
& \ddot{u}_{2}+\omega_{2}^{2} u_{2}=\varepsilon F_{21}+\varepsilon^{2} F_{22}+\sqrt{\varepsilon} G_{2} \dot{\xi}(t),
\end{aligned}
$$

where

$$
F_{1 j}=\frac{f_{2 j}-d_{2} f_{1 j}}{d_{1}-d_{2}}, \quad F_{2 j}=\frac{d_{1} f_{1 j}-f_{2 j}}{d_{1}-d_{2}}, G_{1}=\frac{\sigma_{2}-d_{2} \sigma_{1}}{d_{1}-d_{2}}, G_{2}=\frac{d_{1} \sigma_{1}-\sigma_{2}}{d_{1}-d_{2}}, \quad(j=1,2) .
$$

According to the averaging method the state coordinates $\left(u_{1}, u_{2}\right)$ are to be transformed into the variables $a=\left(a_{1}, a_{2}\right)$ and $\varphi=\left(\varphi_{1}, \varphi_{2}\right)$ by the change

$$
\begin{aligned}
& u_{j}=a_{j} \cos \varphi_{j}, \\
& \dot{u}_{j}=-\omega_{j} a_{j} \sin \varphi_{j} .
\end{aligned}
$$

By using differentiation formula [8] the system of equations (2.4) is transformed into the following system of equations

$$
\begin{aligned}
& \dot{a}_{j}=\varepsilon A_{1 j}(a, \varphi)+\varepsilon^{2} A_{2 j}(a, \varphi)-\sqrt{\varepsilon} G_{j} \frac{\sin \varphi_{j}}{\omega_{j}} \dot{\xi}(t), \\
& \dot{\varphi}_{j}=\omega_{j}+\varepsilon B_{1 j}(a, \varphi)+\varepsilon^{2} B_{2 j}(a, \varphi)-\sqrt{\varepsilon} G_{j} \frac{\cos \varphi_{j}}{a_{j} \omega_{j}} \dot{\xi}(t), \quad(j=1,2),
\end{aligned}
$$

where it is denoted

$$
\begin{aligned}
& A_{1 j}(a, \varphi)=-\frac{F_{j 1}(a, \varphi)}{\omega_{j}} \sin \varphi_{j}+\frac{G_{j}^{2} \cos ^{2} \varphi_{j}}{2 a_{j} \omega_{j}^{2}}, \\
& B_{1 j}(a, \varphi)=-\frac{F_{j 1}(a, \varphi)}{a_{j} \omega_{j}} \cos \varphi_{j}-\frac{G_{j}^{2} \cos \varphi_{j} \sin \varphi_{j}}{a_{j}^{2} \omega_{j}^{2}}, \quad(j=1,2), \\
& A_{2 j}(a, \varphi)=-\frac{F_{j 2}(a, \varphi)}{\omega_{j}} \sin \varphi_{j}, \quad B_{2 j}(a, \varphi)=-\frac{F_{j 2}(a, \varphi)}{a_{j} \omega_{j}} \cos \varphi_{j},
\end{aligned}
$$


The Fokker-Planck (FP) equation for the stationary probability density function $W(a, \varphi)$ takes the form

$$
\sum_{j=1}^{2} \omega_{j} \frac{\partial W}{\partial \varphi_{j}}=-\varepsilon\left[A_{1}, B_{1}\right] L[W]-\varepsilon^{2}\left[A_{2}, B_{2}\right] L[W],
$$

where the operators $\left[A_{j}, B_{j}\right] L[],. j=1,2$ are defined as follows

$$
\begin{aligned}
{\left[A_{1}, B_{1}\right] L[W]=} & \sum_{j=1}^{2}\left[\frac{\partial}{\partial a_{j}}\left(A_{1 j} W\right)+\frac{\partial}{\partial \varphi_{j}}\left(B_{1 j} W\right)\right] \\
& -\sum_{j=1}^{2} \sum_{s=0}^{1}\left\{\frac{\partial^{2}}{\partial a_{j} \partial a_{s}}\left(\frac{G_{j} G_{s} \sin \varphi_{j} \sin \varphi_{s}}{2 \omega_{j} \omega_{s}} W\right)\right. \\
& \left.+\frac{\partial^{2}}{\partial a_{j} \partial \varphi_{s}}\left(\frac{G_{j} G_{s} \sin \varphi_{j} \cos \varphi_{s}}{2 a_{s} \omega_{j} \omega_{s}} W\right)+\frac{\partial^{2}}{\partial \varphi_{j} \partial \varphi_{s}}\left(\frac{G_{j} G_{s} \cos \varphi_{j} \cos \varphi_{s}}{2 a_{j} a_{s} \omega_{j} \omega_{s}} W\right)\right\}
\end{aligned}
$$

$$
\left[A_{2}, B_{2}\right] L[W]=\sum_{j=1}^{2}\left[\frac{\partial}{\partial a_{j}}\left(A_{2 j} W\right)+\frac{\partial}{\partial \varphi_{j}}\left(B_{2 j} W\right)\right] .
$$

We seek the solution of (2.9) in the form

$$
W(a, \varphi)=W_{0}(a, \varphi)+\varepsilon W_{1}(a, \varphi)+\varepsilon^{2} W_{2}(a, \varphi)+\cdots
$$

Substituting (2.11) into (2.9) and comparing the coefficients of like powers of $\varepsilon$ we obtain

$$
\begin{aligned}
\varepsilon^{0}: & \sum_{j=1}^{2} \omega_{j} \frac{\partial W_{0}}{\partial \varphi_{j}}=0 \\
\varepsilon^{1}: & \sum_{j=1}^{2} \omega_{j} \frac{\partial W_{1}}{\partial \varphi_{j}}=-\left[A_{1}, B_{1}\right] L\left[W_{0}\right] \\
\varepsilon^{2}: & \sum_{j=1}^{2} \omega_{j} \frac{\partial W_{2}}{\partial \varphi_{j}}=-\left\{\left[A_{2}, B_{2}\right] L\left[W_{0}\right]+\left[A_{1}, B_{1}\right] L\left[W_{1}\right]\right\}
\end{aligned}
$$

From (2.12) we get

$$
W_{0}=W_{0}(a) .
$$

The arbitrary integration function $W_{0}(a)$ must be chosen from the condition for the function $W_{1}(a, \varphi)$ to be periodic to $\varphi$.

Thus, we get from (2.13)

$$
\left\langle\left[A_{1}, B_{1}\right] L\left[W_{0}(a)\right]\right\rangle=0,
$$


where $\langle$.$\rangle is the averaging operator with respect to \varphi$

$$
\langle.\rangle=\frac{1}{(2 \pi)^{2}} \int_{0}^{2 \pi} \int_{0}^{2 \pi} \cdot(.) d \varphi_{1} d \varphi_{2} .
$$

Substituting (2.10) into (2.16) yields

$$
\sum_{j=1}^{2}\left\{\frac{\partial}{\partial a_{j}}\left(\left\langle A_{1 j}\right\rangle W_{0}(a)\right)-\frac{G_{j}^{2}}{4 \omega_{j}^{2}} \frac{\partial^{2} W_{0}(a)}{\partial a_{j}^{2}}\right\}=0 .
$$

The second term $W_{1}(a, \varphi)$ in (2.11) is determined from (2.13), using Fourier expansion

$$
\left[A_{1}, B_{1}\right] L\left[W_{0}(a)\right]=W_{0}(a) \sum_{k_{1}} \sum_{k_{2}} C_{k_{1} k_{2}}(a) \exp \left[i\left(k_{1} \varphi_{1}+k_{2} \varphi_{2}\right)\right]
$$

where

$$
C_{k_{0} k_{1}}(a)=\frac{1}{(2 \pi)^{2} W_{0}(a)} \int_{0}^{2 \pi} \int_{0}^{2 \pi}\left[A_{1}, B_{1}\right] L\left[W_{0}(a)\right] \exp \left[-i\left(k_{0} \varphi_{0}+k_{1} \varphi_{1}\right)\right] d \varphi_{0} d \varphi_{1} .
$$

Sustituting (2.19) into (2.13) yields

$$
W_{1}(a, \varphi)=W_{0}(a)\left[W_{10}(a)+\sum_{k_{1}} \sum_{k_{2}} \frac{C_{k_{1} k_{2}}(a)}{k_{1} \omega_{1}+k_{2} \omega_{2}} \exp \left[i\left(k_{1} \varphi_{1}+k_{2} \varphi_{2}\right)\right]\right]
$$

where

$$
k_{1} \omega_{1}+k_{2} \omega_{2} \neq 0 .
$$

The arbitrary integration function $W_{10}(a)$ must be chosen from the condition for the function $W_{2}(a, \varphi)$ to be periodic to $\varphi$. Similarly, we can find the third term $W_{2}(a, \varphi)$ in (2.11).

\section{Application}

In order to illustrate the procedure proposed we consider the system with cubic damping whose equation of motion takes the form

$$
\begin{aligned}
& \ddot{x}_{1}+c_{11} x_{1}-c_{22} x_{2}=-2 \varepsilon\left(h_{11} \dot{x}_{1}-h_{12} \dot{x}_{2}\right)-\varepsilon^{2} \beta \dot{x}_{1}^{3}+\sqrt{\varepsilon} \sigma_{1} \dot{\xi}(t), \\
& \ddot{x}_{2}-c_{22} x_{1}+c_{22} x_{2}=-2 \varepsilon h_{12}\left(\dot{x}_{2}-\dot{x}_{1}\right)+\sqrt{\varepsilon} \sigma_{2} \dot{\xi}(t) .
\end{aligned}
$$

The physical model of this system is represented in Fig. 1 . Where $k_{11}, k_{12}, h_{11}, h_{12}, \beta, \sigma_{1}$, $\sigma_{2}$ are posicive constants, $m_{1}=m_{2}=1$ and

$$
\begin{aligned}
& c_{11}=k_{11}+k_{12}, \quad c_{22}=k_{12}, \quad h_{11}=h_{1}+h_{12}, \\
& R_{1}=-\varepsilon^{2} \beta \dot{x}_{1}^{2}+\sqrt{\varepsilon} \sigma_{1} \dot{\xi}(t), \quad R_{2}=\sqrt{\varepsilon} \sigma_{2} \dot{\xi}(t) .
\end{aligned}
$$




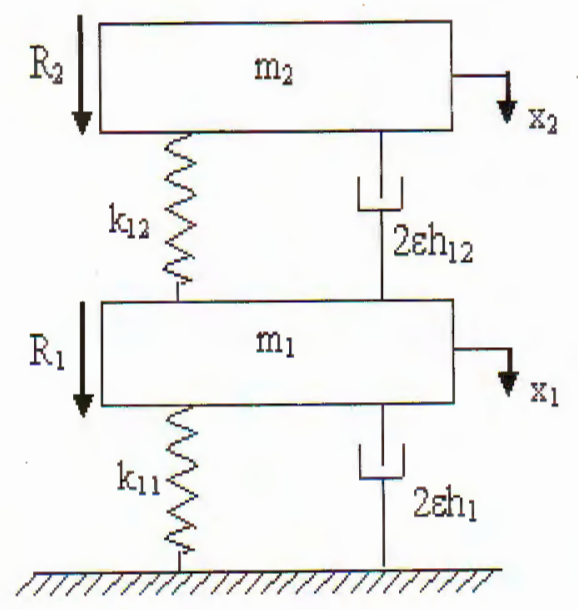

Fig. 1

In this case we have

$$
\begin{aligned}
& f_{11}=-2 h_{11} \dot{x}_{1}+2 h_{12} \dot{x}_{2}, \quad f_{12}=-\beta \dot{x}_{1}^{3}, \\
& f_{21}=2 h_{21} \dot{x}_{1}-2 h_{12} \dot{x}_{2}, \quad f_{22}=0 \\
& \omega_{1,2}^{2}=\frac{c_{11}+c_{22} \mp \sqrt{\left(c_{11}-c_{22}\right)^{2}+4 c_{22}^{2}}}{2}, \\
& d_{1,2}=\frac{c_{11}-c_{22}}{2 c_{22}} \pm \frac{\sqrt{\triangle}}{2 c_{22}} \\
& d_{1}-d_{2}=\frac{\sqrt{\triangle}}{c_{22}}, \quad \triangle=\left(c_{11}-c_{22}\right)^{2}+4 c_{22}^{2} .
\end{aligned}
$$

Substituting (3.3) into (2.5) yields

$$
\begin{aligned}
& F_{11}=p_{11} \dot{x}_{1}+p_{12} \dot{x}_{2}, \quad F_{12}=p_{13} \dot{x}_{1}^{3}, \\
& F_{21}=p_{21} \dot{x}_{1}+p_{22} \dot{x}_{2}, \quad F_{22}=p_{23} \dot{x}_{1}^{3},
\end{aligned}
$$

where

$$
\begin{aligned}
& p_{11}=\frac{2\left(h_{12}+d_{2} h_{11}\right)}{d_{1}-d_{2}}, \quad p_{12}=\frac{-2 h_{12}\left(1+d_{2}\right)}{d_{1}-d_{2}}, \quad p_{13}=\frac{d_{2} \beta}{d_{1}-d_{2}}, \\
& p_{21}=\frac{2\left(d_{1} h_{11}+h_{12}\right)}{d_{1}-d_{2}}, \quad p_{22}=\frac{2 h_{12}\left(1+d_{2}\right)}{d_{1}-d_{2}}, \quad p_{23}=-\frac{d_{1} \beta}{d_{1}-d_{2}}
\end{aligned}
$$

From (2.8), using (2.2), (2.6) and (3.4), after calculations we obtain

$$
\begin{aligned}
& \left\langle A_{11}\right\rangle=\frac{p_{11}+p_{12} d_{1}}{2} a_{1}+\frac{G_{1}^{2}}{4 \omega_{1}^{2} a_{1}}, \quad\left\langle A_{12}\right\rangle=\frac{p_{21}+p_{22} d_{2}}{2} a_{2}+\frac{G_{2}^{2}}{4 \omega_{2}^{2} a_{2}}, \\
& \left\langle A_{21}\right\rangle=\frac{3 p_{13}}{8}\left(\omega_{1}^{2} a_{1}^{3}+2 \omega_{2}^{2} a_{1} a_{2}^{2}\right), \quad\left\langle A_{22}\right\rangle=\frac{3 p_{23}}{8}\left(2 \omega_{1}^{2} a_{1}^{2} a_{2}+\omega_{2}^{2} a_{2}^{3}\right) .
\end{aligned}
$$


Substituting (3.6) into (2.18) we have

$$
W_{0}(a)=C a_{1} a_{2} \exp \left[\frac{\omega_{1}^{2}}{G_{1}^{2}}\left(p_{11}+p_{12} d_{1}\right) a_{1}^{2}+\frac{\omega_{2}^{2}}{G_{2}^{2}}\left(p_{21}+p_{22} d_{2}\right) a_{2}^{2}\right] .
$$

The parameters in the expression of $W_{0}(a)$ must satisfy the inequalities

$$
\gamma_{1}=\frac{\omega_{1}^{2}}{G_{1}^{2}}\left(p_{11}+p_{12} d_{1}\right)<0, \quad \gamma_{2}=\frac{\omega_{2}^{2}}{G_{2}^{2}}\left(p_{21}+p_{22} d_{2}\right)<0 .
$$
obtain

Substituting (3.8) into (2.13), using (2.6), (2.8) and (3.5), after some calculations we

$$
\begin{aligned}
W_{11}(a, \varphi)= & \frac{p_{11}+p_{12} d_{1}}{2 \omega_{1}} \sin 2 \varphi_{1}+\frac{p_{21}+p_{22} d_{2}}{2 \omega_{2}} \sin 2 \varphi_{2} \\
& +\frac{1}{\omega_{1}^{2}-\omega_{2}^{2}}\left(\omega_{2} s_{12} a_{1} a_{2}-\frac{G_{1} G_{2}}{\omega_{1}} \frac{1}{a_{1} a_{2}}\right) \sin \varphi_{1} \cos \varphi_{2} \\
& +\frac{1}{\omega_{1}^{2}-\omega_{2}^{2}}\left(\frac{G_{1} G_{2}}{\omega_{2}} \frac{1}{a_{1} a_{2}}-\omega_{1} s_{12} a_{1} a_{2}\right) \cos \varphi_{1} \sin \varphi_{2}
\end{aligned}
$$

where

$$
\begin{aligned}
s_{12}= & \frac{2 \omega_{1} \omega_{2}}{G_{1}^{2} G_{2}^{2}}\left[G_{2}^{2}\left(p_{11}+d_{1} p_{12}\right)\left(p_{11}+d_{2} p_{12}\right)+G_{1}^{2}\left(p_{21}+d_{1} p_{22}\right)\left(p_{21}+d_{2} p_{22}\right)\right. \\
& \left.-2 \omega_{1} \omega_{2}\left(p_{11}+d_{1} p_{12}\right)\left(p_{21}+d_{2} p_{22}\right)\right] .
\end{aligned}
$$

Substituting (3.8) and (3.11) into (2.14) we have the equation for the arbitrary function $W_{10}(a)$ in the form

$$
\sum_{j=1}^{2}\left\{\frac{\partial}{\partial a_{j}}\left[\left\langle A_{1 j}\right\rangle W_{0}(a) W_{10}(a)\right]-\frac{G_{j}^{2}}{4 \omega_{j}^{2}} \frac{\partial^{2}}{\partial a_{j}^{2}}\left[W_{0}(a) W_{10}(a)\right]\right\}=-\sum_{j=1}^{2} \frac{\partial}{\partial a_{j}}\left[<A_{2 j}>W_{0}(a)\right] .
$$

From (3.12), using (3.6) and (3.7) we have

$$
W_{10}(a)=\alpha_{1} a_{1}^{2}+\alpha_{2} a_{2}^{2}+\alpha_{12} a_{1}^{2} a_{2}^{2}+\alpha_{11} a_{1}^{4}+\alpha_{22} a_{2}^{4},
$$

where

$$
\begin{aligned}
& \alpha_{1}=-\frac{3 \omega_{1}^{2} d_{1} \beta}{2\left(d_{1}-d_{2}\right) \gamma_{1}}, \quad \alpha_{2}=-\frac{3 \omega_{2}^{2} d_{2} \beta}{2\left(d_{1}-d_{2}\right) \gamma_{2}}, \\
& \alpha_{11}=\frac{3 \omega_{1}^{4} d_{2}\left(d_{1}-d_{2}\right) \beta}{8\left(\sigma_{2}-d_{2} \sigma_{1}\right)^{2}}, \quad \alpha_{22}=-\frac{3 \omega_{2}^{4} d_{1}\left(d_{1}-d_{2}\right) \beta}{8\left(\sigma_{2}-d_{1} \sigma_{1}\right)^{2}}, \\
& \alpha_{12}=\frac{3 \omega_{1}^{2} \omega_{2}^{2} \beta\left(d_{2} G_{2}^{2} \gamma_{1}-d_{1} G_{1}^{2} \gamma_{2}\right)}{2 G_{1}^{2} G_{2}^{2}\left(\gamma_{1}+\gamma_{2}\right)} .
\end{aligned}
$$

Thus, the second order approximate solution of the FP equation (2.9) for the system (3.1) takes the form

$$
W(a, \varphi)=W_{0}(a)\left\{1+\varepsilon\left[W_{10}(a)+W_{11}(a, \varphi)\right]\right\} .
$$


where $W_{0}(a), W_{11}(a)$ and $W_{10}(a)$ are defined in (3.8), (3.10) and (3.13), respectively. It is seen from (3.13) and (3.1) that the effect of the nonlinear term $\varepsilon^{2} \beta \dot{x}^{3}$ is shown in (3.13) and (3.14).

The approximate mean squares $E\left[x_{1}^{2}\right]$ and $E\left[x_{2}^{2}\right]$ are to be found

$$
E\left[x_{i}^{2}\right]=\int_{0}^{2 \pi} \int_{0}^{2 \pi} \int_{0}^{\infty} \int_{0}^{\infty} x_{i}^{2} W(a, \varphi) d a_{1} d a_{2} d \varphi_{1} d \varphi_{2}, \quad(i=1,2) .
$$

Substituting (3.15) and $x_{i}$ in (2.2), (2.6) into (3.16), after calculations we have

$$
\begin{aligned}
E\left[x_{1}^{2}\right]= & -\frac{\gamma_{1}+\gamma_{2}}{\gamma_{1} \gamma_{2}}+\varepsilon \frac{2}{\gamma_{1}^{3} \gamma_{2}^{3}}\left[\alpha_{1} \gamma_{1} \gamma_{2}^{3}+\alpha_{2} \gamma_{1}^{3} \gamma_{2}-3 \alpha_{12} \gamma_{1} \gamma_{2}\left(\gamma_{1}+\gamma_{2}\right)\right. \\
& \left.+2 \alpha_{11}\left(\gamma_{1}^{3}+\gamma_{1}^{2} \gamma_{2}-2 \gamma_{1} \gamma_{2}^{2}-6 \gamma_{2}^{3}\right)+2 \alpha_{22}\left(\gamma_{2}^{3}+\gamma_{2}^{2} \gamma_{1}-2 \gamma_{2} \gamma_{1}^{2}-6 \gamma_{1}^{3}\right)\right] \\
E\left[x_{2}^{2}\right]= & -\frac{d_{1}^{2} \gamma_{2}+d_{2}^{2} \gamma_{1}}{\gamma_{1} \gamma_{2}}+\varepsilon \frac{2}{\gamma_{1}^{3} \gamma_{2}^{3}}\left[\alpha_{1} d_{1}^{2} \gamma_{1} \gamma_{2}^{3}+\alpha_{2} d_{2}^{2} \gamma_{1}^{3} \gamma_{2}-3 \alpha_{12} \gamma_{1} \gamma_{2}\left(d_{1}^{2} \gamma_{1}+d_{2}^{2} \gamma_{2}\right)\right. \\
& +2 \alpha_{11}\left(d_{2}^{2} \gamma_{1}^{3}+d_{1}^{2} \gamma_{1}^{2} \gamma_{2}-2 d_{2}^{2} \gamma_{1} \gamma_{2}^{2}-6 d_{1}^{3} \gamma_{2}^{3}\right) \\
& \left.+2 \alpha_{22}\left(d_{1}^{2} \gamma_{2}^{3}+d_{2}^{2} \gamma_{2}^{2} \gamma_{1}-2 d_{1}^{2} \gamma_{2} \gamma_{1}^{2}-6 d_{2}^{2} \gamma_{1}^{3}\right)\right]
\end{aligned}
$$

In the case $\beta=0$ (linear system) we have

$$
E\left[x_{1}^{2}\right]=-\frac{\gamma_{1}+\gamma_{2}}{\gamma_{1} \gamma_{2}}, \quad E\left[x_{2}^{2}\right]=-\frac{d_{1}^{2} \gamma_{1}+d_{2}^{2} \gamma_{2}}{\gamma_{1} \gamma_{2}} .
$$

\section{Conclusion}

For many years the stochastic averaging method has been a very useful tool for investigating non-linear vibration systems subject to white noise and coloured noise excitations. In this paper, the higher order stochastic averaging method is applied to non-linear systems with two degrees of freedom subject to white noise excitations. The application to the system with cubic damping is considered and shows the effect of the non-linear term to the mean square response of the system.

Acknowledgement. Support from the Council for natural sciences of Vietnam is gratefully acknowledged.

\section{References}

1. Anh N. D., Higher order approximate solutions in stochastic averaging method. In Proc. Of NCSR of VN 5 (1993) 19-26.

2. Anh N. D., Higher order averaging method of coefficients in Fokker-Planck equation. In special volume: Advances in Nonlinear Structural Dynamics of SADHANA, Indian Acad. Of Sc. (1995) 373-388.

3. Anh N. D. and Tinh N. D., Higher order averaging solutions for Van-Der-Pol oscillator. In Proc. Int. Conf. On Nonlinear Stochastic Dynamics, Hanoi, Vietnam (1995) $27-38$.

4. Anh N. D. and Tinh N. D., The influence of second order narrow-band colored noises on non-linear random vibrations. In Vietnam Journal of Mechanics, NCST of Vietnam. 21 (1999) 65-74. 
5. Ariaratnam S. T., Tam D. S. F., Random vibration and stability of a linear parametrically exited oscillator. Z. Angrew. Math. Mech. 59 (1979) 79-84.

6. Bolotin V. V., Random Vibration of Elastic Systems, Haygue: Martinus Nijhoff, 1984.

7. Ibrahim R. A., Parametric Random Vibration, (Hertfordsire New York: Research Studies Press/John Wiley and Sons) 1985.

8. Mitropolskii Iu. A., Dao N. V., Anh N. D., Nonlinear Oscillations in Systems of Arbitrary Order, Naukova-Dumka, Kiev, 1992 (In Russian).

9. Red-Horse J. R., Spanos P. D., A generalization to stochastic averaging in random vibration, Int. J. Nonlinear Mech. 27 (1992) 85-101.

10. Roberts J. B., Spanos P. T. D., Stochastic averaging: An approximate method of solving random vibration problem, Int. J. Nonlinear Mech. 21 (1986) 111-134.

11. Tinh N. D., Higher Order Stochastic Averaging Method in Nonlinear Vibration Systems, Doctorate Diploma, Hanoi, 1999 (In Vietnamese).

12. Zhu W. Q., Stochastic averaging method in random vibrations. Appl. Mech. Rev. 41 (1988) 189-199.

13. Zhu W. Q., Yu M. Q., Lin Y. K., On improved stochastic averaging procedure, Probab. Eng. Mech. 9 (1994) 203-212.

14. Zhu W. Q. et al., Stochastic averaging of quasi - integrable Hamiltonian systems, J. Applied Mech. 64 (1997) 975-984.

Received May 14, 2003

\section{PHUONG PHÁP TRUNG BİNH NGẪU NHIÊN BẬC CAO ĐỐI VỚI HỆ HAI BẬC TỰ DO}

Phương pháp trung bình ngẫu nhiên bậc cao đã được áp dụng rộng rãi đối với các hệ dao động phi tuyến một bậc tự do chịu kích động ngẫu nhiên dạng ồn trắng và ồn màu. Trong bài báo này, phương pháp tiếp tục được trình bày đối với các hệ phi tuyến yếu hai bậc tự do chịu kích động ngẫu nhiên dạng ồn trắng. Sau đó phương pháp được áp dụng để xác định nghiệm xấp xỉ bậc hai của phương trình Fokker-Planck đối với hệ có càn phi tuyến bậc ba. 